\title{
Introduction of a New Baffle-Plate Concept to Control the Gas Flow Behavior in a Large Volume PECVD Chamber
}

\author{
Gustavo Simiema de Freitas Barbosa1,2, Klaus Vissing1, Bernd Mayer² \\ ${ }^{1}$ Fraunhofer Institute for Manufacturing Technology and Advanced Materials (IFAM), Wiener Straße 12, Bremen, Germany \\ ${ }^{2}$ University of Bremen, Faculty of Production Engineering, Badgasteiner Straße 1, Bremen, Germany \\ Email: gustavo.barbosa@ifam.fraunhofer.de
}

How to cite this paper: de Freitas Barbosa, G.S., Vissing, K. and Mayer, B. (2018) Introduction of a New Baffle-Plate Concept to Control the Gas Flow Behavior in a Large Volume PECVD Chamber. Open Journal of Fluid Dynamics, 8, 141-160. https://doi.org/10.4236/ojfd.2018.82011

Received: April 4, 2018

Accepted: June 8, 2018

Published: June 11, 2018

Copyright $\odot 2018$ by authors and Scientific Research Publishing Inc. This work is licensed under the Creative Commons Attribution International License (CC BY 4.0).

http://creativecommons.org/licenses/by/4.0/

\begin{abstract}
Achieving a reasonable homogeneity of the coating deposition rate within a low-pressure plasma process is a challenge, especially in large volume chambers. The local gas flow behavior is one key parameter in the coating deposition. Basically, with the exception of the product geometry and the electrode design, there are two main influences on the gas flow distribution inside a large volume chamber: 1) gas feed-in system and 2) gas exhaustion system. This work focuses on the gas exhaustion system with the aim to reduce its influence on the gas flow behavior inside a large plasma coater. In this sense, a solution with a perforated plate, named "Baffle-Plate", is created. Thereby relevant construction parameters are identified and investigated to understand their influence in respect to the homogeneity of the gas exhaustion. Number of holes, hole diameter, distance of the Baffle-Plate to the top of the chamber, gas flow and chamber volume are evaluated parameters. Computational fluid dynamics (CFD) simulations are used as a tool to determine velocity and pressure distribution inside the PECVD-chamber and, consequently, to evaluate the layout parameters of the Baffle-Plate. Additionally, practical coating experiments with and without the Baffle-Plate installed are performed. The results show a correlation between the gas flow distribution and the homogeneity of the coating deposition rate. With these results construction guidelines have been formulated. Hence in future developments correct technical layouts of the Baffle-Plate can be applied, easily.
\end{abstract}

\section{Keywords}

PECVD, Plasma Polymerization, Coating Homogeneity, Gas Velocity, Computation Fluid Dynamics 


\section{Introduction}

The formation of functional thin film coatings can be achieved through different processes, such as spin coating, chemical vapor deposition, sputtering and plasma enhanced chemical vapor deposition (PECVD) [1]. PECVD (plasma polymerization is another often used term) is a technique widely used for the modification of surface properties of different materials, e.g., metals, polymers and composites [2]. For instance, tailored coatings permit the enhancement of mechanical, chemical, electrical and optical properties of surfaces [3] [4] [5] [6] [7]. Therefore this technique is very useful for different ranges of industrial fields, such as aircraft, aerospace, automotive, packaging, food, textile and medical devices [8] [9] [10].

Concentrating on the PECVD process, the substrate is placed on a low-pressure chamber supplied with precursor gases and subjected to the effects of the plasma. Due to the plasma, the precursor gases are fragmented and became able to react and form a thin film coating onto the surface of the substrate as well as in every surface positioned inside or near the plasma region [5]. Besides plasma parameters like pressure, gas flow and power, for these reactions to occur a certain time is necessary. Therefore, it is very important to control the gas velocity inside the plasma chamber to ensure homogenous coating properties. The management of the gas flow inside the chamber is possible through the control of the gas feed-in and gas exhaustion system [11] [12]. The domain over the gas flow behavior is even more challenger when the scaling up is desired whether to process many products in one batch or to coat one part with large dimensions. In this perspective, the design of the plasma chamber is crucial to achieve homogeneous deposition rates as well as good quality of the formed thin film. Hence, the gas exhaustion system, gas feed-in system and the electrodes must be carefully designed. From the state of the art, there are some patents issued regarding gas distribution systems for small plasma chambers in the context of semiconductor industry [11] [12] [13] [14]. However, concerning large volume plasma chambers, normally installed in other industries, there is limited information either due to lack of knowledge or due to confidential issues. Especially for these cases, which the improvement of the cost effectiveness is very important, is expected that all the products processed in one batch show the same surface properties and also a homogeneous coating thickness distribution. Hence, the local gas flow behavior is one key feature to enable the scaling up of plasma polymerization processes. Basically, with the exception of the product geometry and the electrode design, there are two main influences on the gas flow distribution inside a large volume chamber: 1) gas feed-in system and 2) gas exhaustion system. They must be carefully designed to guarantee an evenly gas flow through the products and consequently accomplish reasonable coating properties.

In our previous work [15] the gas flow behavior inside the gas feed-in system was investigated, using CFD tool as support, varying some construction layouts, 
e.g. number of holes, hole diameter, pipe section area, pipe length and pipe aspect ratio. Afterwards construction guidelines were developed. It was shown that the pipe section area is the most important parameter to reach an even gas distribution. The investigation showed that the pipe section area must be bigger than $20 \mathrm{~mm} \times 20 \mathrm{~mm}$ to achieve a homogeneous gas supply through the pipe holes.

In comparison to that this work focuses on the gas exhaustion system and the main objective is to investigate its influence on the gas flow distribution inside the current setup of the low-pressure plasma chamber with $1 \mathrm{~m}^{3}$ volume. Based on the outcomes, a solution to reduce the influence of the big and central gas exhaustion system is described. Computational fluid dynamics (CFD) simulations are used to investigate the gas velocity and pressure inside the PECVD chamber. The simulation results are used to correlate the calculated gas velocity with the coating thickness distribution obtained through experiments.

\subsection{Description of the Low-Pressure Plasma Chamber}

A large volume plasma reactor, about $1 \mathrm{~m}^{3}$ volume, developed by Fraunhofer IFAM with symmetric electrode configurations is used. It is operated with a capacitive radio-frequency generator $(13.56 \mathrm{MHz})$. In Figure 1, the used plasma system setup is shown schematically, XY Plane view, with its most important components. Basically, it has a "U-form" electrode, represented by the blue geometry, connected to the RF-Generator. To keep the symmetry, the "U-form" grounded counter electrode is formed by the door, the back wall and the perforated grid. This grid has also a protection function: avoid plasma to burn inside the exhaustion system and therefore to preserve the integrity of the chamber.

To regulate the process pressure, which is $0.015 \mathrm{hPa}$, an adjustable butterfly valve is installed in the gas exhaustion. During the process, gas is continuously introduced via the gas feed-in system and the butterfly valve ensures a constant

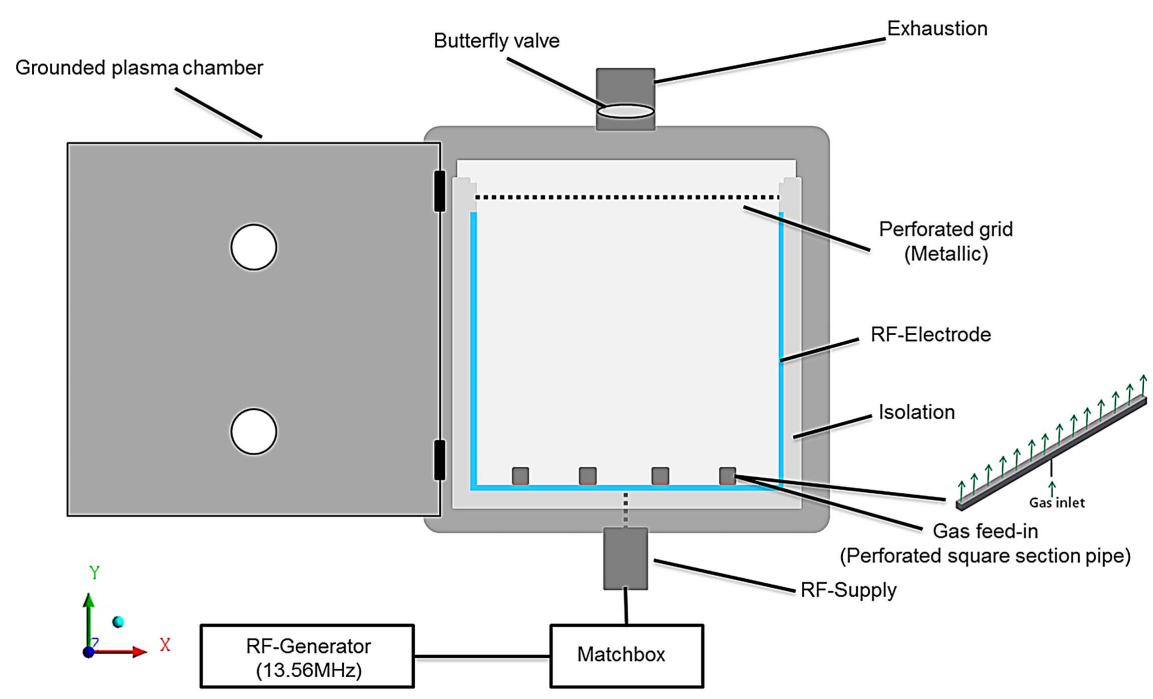

Figure 1. Sketch of the low-pressure plasma chamber. 
process pressure together with the exhaustion system. The power is generated by the RF-generator, coupled via the Matchbox with the electrode. The Matchbox works to compensate the unsteady ohmic resistance of the plasma and at the same time, the RF-generator is protected against backscattered power.

\subsection{Influence Zone: Gas Exhaustion System and Gas Feed-In System}

From our previous work, Figure 2 shows the YZ Plane view of the gas simulation result from the current setup of the low-pressure plasma chamber. The simulation conditions are as shown in Table 1. The gas feed-in distributor consists of a rectangular pipe closed at both ends. At the bottom of the gas feed-in distributor the gas inlet is centrally placed. The rectangular pipe contains equally spaced perforations on the top surface to provide the gas supply into the PECVD chamber. More details of the construction can be seen in our previous work [15]. The influence zone of the gas feed-in system represents a small fraction of the plasma chamber volume. The gradient color, which means velocity gradient, extends over approximately $5-10 \mathrm{~cm}$ from the pipe holes. Nevertheless, it is very important to have it in mind before an experiment or before a complete construction project of a low-pressure plasma chamber is done. From the gas exhaustion system point of view, its influence zone is much larger than the one from the gas feed-in system. The gradient color is much more intense in the region of the exhaustion system in comparison to the gas supply region. Having this picture in mind, in a coating process is expected to see a similar pattern which would represent a gradient in the deposition rate.

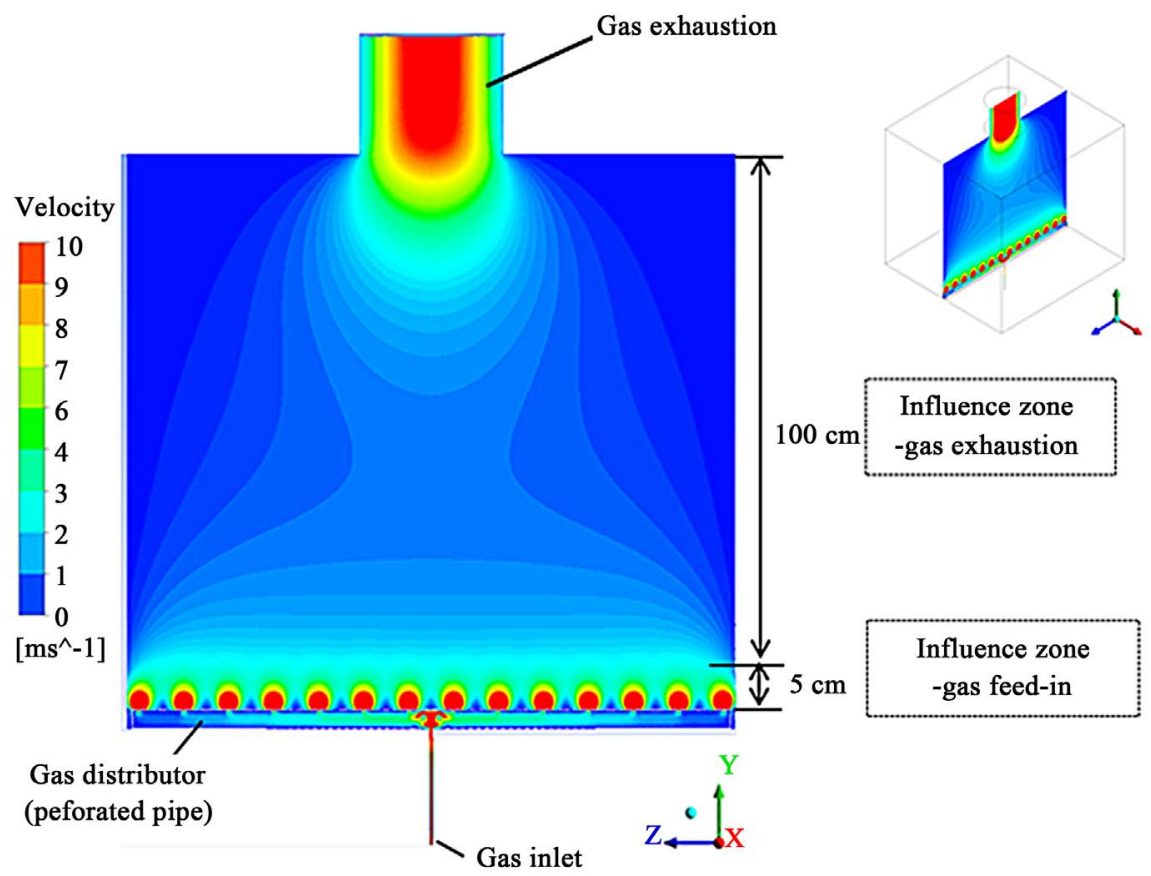

Figure 2. Gas simulation result showing the two dissimilar influence zones of the gas feed-in and of the gas exhaustion. Adapted from [15]. 
In this sense, this work is divided in two parts. Part I refers to the characterization of the current condition of the plasma-coater through gas flow simulation (2.1.1) and experiment (2.1.2). Part II refers to the description of the solution to minimize the influence of the gas exhaustion system in the gas flow distribution inside the plasma coater and consequently in the local coating deposition rate. In the same way as in Part I, gas flow simulations (3.1.1) and experiments (3.1.2) are performed. Afterwards, construction guidelines for this solution is listed.

\section{Part I: Current Condition}

\subsection{Methods}

\subsubsection{CFD Simulations}

The CFD simulations were performed applying the software package Ansys Fluent $16.0^{\circledR}$. A quarter of the model was used. It allows shorter simulation time and mesh refinement at important regions (gas feed-in holes and gas exhaustion region). Moreover, they require relatively less modelling effort (e.g. pre-processing) when compared to a full model analysis.

Figure 3(a) and Figure 3(b) show the full model of the experiment setup. Part c of Figure 3 shows the quarter of the experiment setup model, which was used for the gas flow simulation. It can be seen that the metallic grounded perforated grid (Figure 1) was not included in the gas simulation due to process limitation of the computer used. As the name already suggests, perforated grid, is a metallic grid completely perforated with $1 \mathrm{~mm}$ diameter holes in sequence. It means that by adding the perforated plate to the simulation the number of elements to be calculated would jump from less than $10^{6}$ to more than $20^{6}$ which makes unfeasible the calculation.

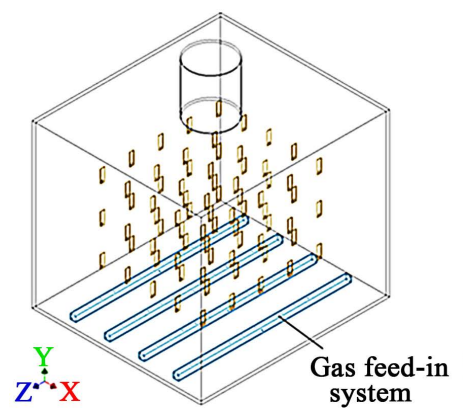

(a)

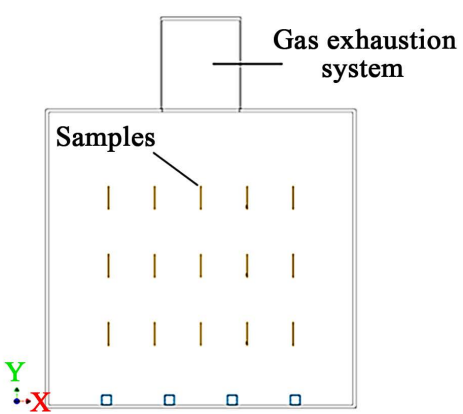

(b)

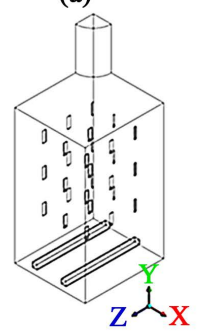

(c)

Figure 3. Model of the experiment setup (a) Isometric view; (b) Front view; (c) isometric view of a quarter of the model. 
For all CFD simulations carried out the boundary conditions were defined to reproduce the conditions of the experiment (Table 1). The number of elements in each model was approximately 3,000,000. Grid independence tests were performed to determine the greatest mesh refinement. The solutions were converged when all the residuals were smaller than $10^{-5}$ for the continuity and momentum equations and smaller than $10^{-6}$ for the energy equation.

\subsubsection{Experiment}

To verify the current condition of the low-pressure plasma-chamber regarding the deposition rate distribution, an experiment was done, as shown in Figure 4. The experiment consists in coating $\mathrm{Si}$-Wafers equally distributed in the volume of the chamber and in floating potential (i.e. the samples were not direct in contact with the electrodes). To perform it, the Si-wafers were fixed on a sheet of glass (Figure 4) and this assembly was fixed to a nylon wire and hanged at the perforated grid. A total of 25 nylon wires were used, each one with three assemblies of Si-wafers + Glass equally spaced. As shown in Figure 4 there is a " $U$ " form electrode system, which is connected to the RF-generator and the other " $U$ " is formed with the grounded electrodes. Hence, the area ratio of powered electrode to grounded electrode is equal one, which means a Self-Bias free process.
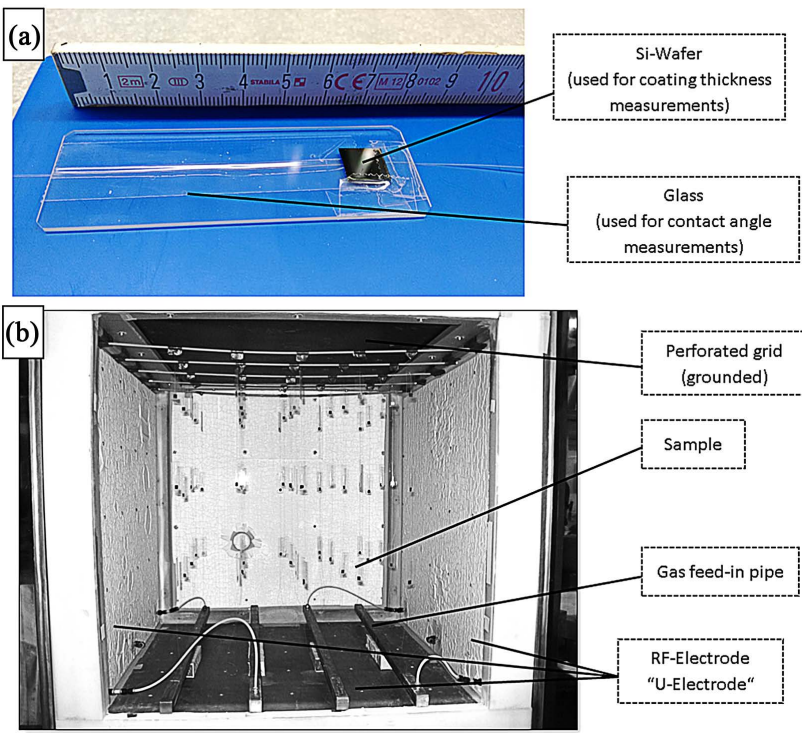

Figure 4. (a) Samples used in the experiment; (b) Experimental setup.

Table 1. Boundary conditions for the gas flow simulation of the experiment.

\begin{tabular}{ccc}
\hline Model & Boundary conditions & Values \\
\hline Laminar flow with low pressure & Gas Temperature & $298.15[\mathrm{~K}]$ \\
boundary slip & Wall Temperature & $298.15[\mathrm{~K}]$ \\
Ideal gas & Mass flow rate of $\mathrm{N}_{2}$ & $2.76 \mathrm{E}-06[\mathrm{~kg} / \mathrm{s}]$ \\
Steady state & Pressure (outlet) & $0.015[\mathrm{hPa}]$
\end{tabular}


The coating process used under the frame of all experiments in this work is-

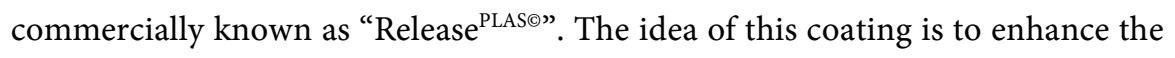
processes where a mold is needed or applications where a barrier (low surface energy) to attachments (i.e. dirty, insects) is desired [16]. Current industrial applications which use carbon fibers, especially for the aircraft industry, release agents are applied in the mold surface before the lamination. Due to this release agent application, additional steps are required in the production process. The mold must be cleaned before other lamination can be started and also the demolded product must be cleaned before moving to the next stage. Either the mold or the parts can be damaged during the demolding process. This is a drawback in a market where the costs must be minimized. In this context, a coating with "release" properties is very welcome. The Release ${ }^{\text {PLAS@ }}$ coating shows the following advantages:

- Protect the mold and the molded part

- Facilitate the demolding of the part

- Chemically and thermal stable

- Avoid additional processing steps on the mold and on the molded part

To realize such a polymeric coating, with low polar part, a Self-Bias free process is necessary [17]. Otherwise, the ion bombardment on the surface of the mold to be coated would be very intense, due to a negative Bias potential. More methyl components would be broken in small products and exhausted from the vacuum pump. It leads to a more $\mathrm{SiO}_{\mathrm{x}}$ characteristic coating, losing the "release" properties.

\subsection{Results and Discussion}

\subsubsection{CFD Simulations}

The results from the gas flow simulation of the experiment setup are depicted in Figure 5, where part "a" represents the top plane, part "b" middle plane and part "c" bottom plane. To make the results more visual, the simulation result for the quarter model was mirrored in the two symmetry planes. One can observe in Figure 5, that the gas obtains high velocity in the middle, represented by the red color, and low velocity near to the walls represented by the dark blue color. It is also obvious, that the gas velocity increases toward the gas exhaustion system, from bottom plane to top plane. Additionally, the samples influenced the gas flow inside the chamber characterized by the lower velocities closed to them in comparison to the neighbor region.

\subsubsection{Experiment}

Figure 6 shows the results of the calculated deposition rate based on the measurements of the coating thickness distribution over the Si-Wafers and interpolated using the software Origin. The deposition rate was calculated dividing the measured coating thickness by the coating deposition time.

The calculated deposition rate was plotted using a color contour graphic. The used kind of plot makes the understanding easier in a way that a $3 \mathrm{D}$ experiment 


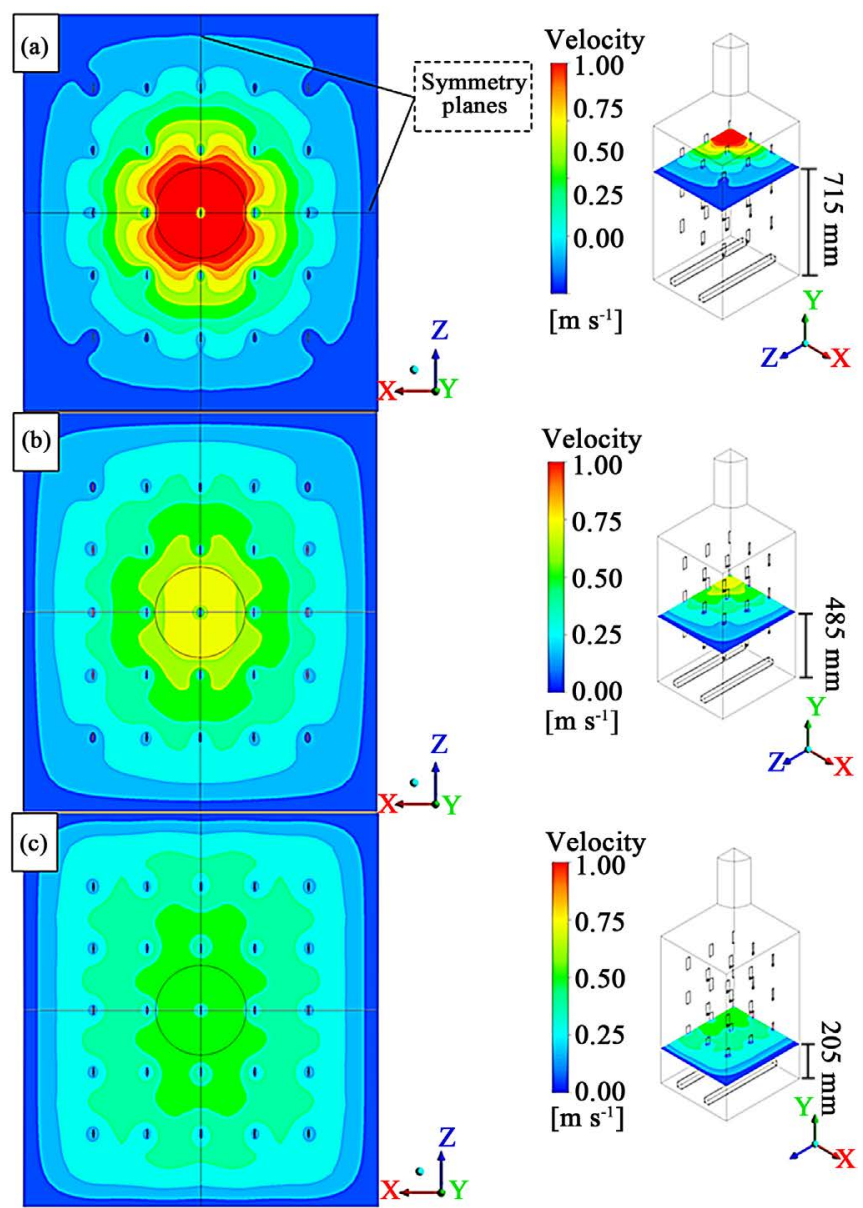

Figure 5. Gas simulation results of the experiment-Current setup.

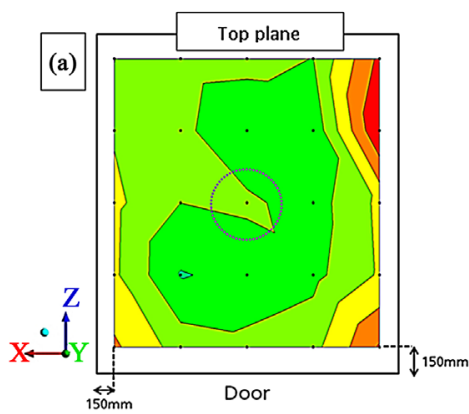

(b)

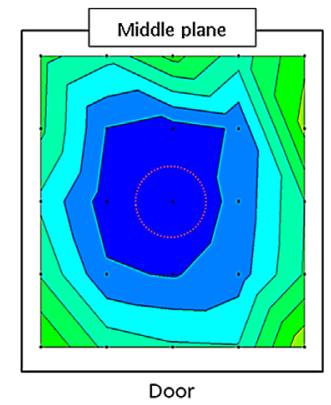

(c)

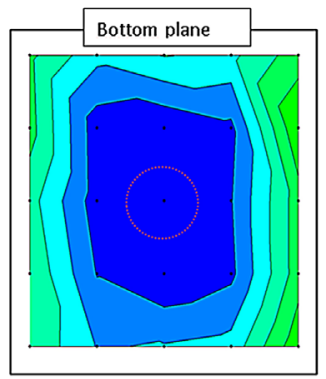

Door
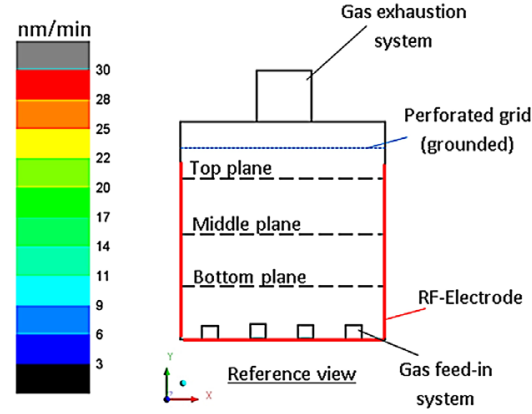

Figure 6. Coating deposition rate distribution (a) top plane; (b) middle plane; (c) bottom plane. 
is shown in a $2 \mathrm{D}$ graphic. In this case, low deposition rate and high deposition rate are represented in blue and red, respectively.

The results are shown again in three different planes: top plane (Figure 6(a)), middle plane (Figure 6(b)) and bottom plane (Figure 6(c)). Each plane contains 25 samples. Their positions are represented by the black points in each image.

In all three planes (bottom, middle and top plane), high deposition rates on the edges (close to the electrodes) and low deposition rates in the middle were observed. These results suggest that there is a non-homogeneous gas flow inside the chamber. Additionally, Figure 7 shows clearly that the deposition rate increases from bottom plane to the top plane while in the bottom and middle plane was practically the same. Furthermore, very high standard deviations can be observed, especially in the bottom and middle plane. Since there is no literature available to compare these values, as coating deposition is process and chamber dependent, the values obtained are the reference starting points for new improvements in the standard low-pressure plasma chamber focusing on reducing the inhomogeneity $(\Phi)$. In this experiment, the top plane shows the lowest inhomogeneity calculated by the following equation:

$$
\Phi(\%)=\frac{\text { Standard Deviation of deposition rate }}{\text { Average of deposition rate }}
$$

Based on the experimental and on the gas simulation results the following hypotheses are formulated:

- The gas exhaustion system is influencing the coating deposition rate.

- Higher velocities (in the chamber middle) lead to lower deposition rates and lower velocities (near to the walls) lead to higher deposition rates (with other precursor gases there might be differences). Nevertheless, this contemplation cannot explain why the top plane show higher deposition rates because it shows in average a higher gas velocity.

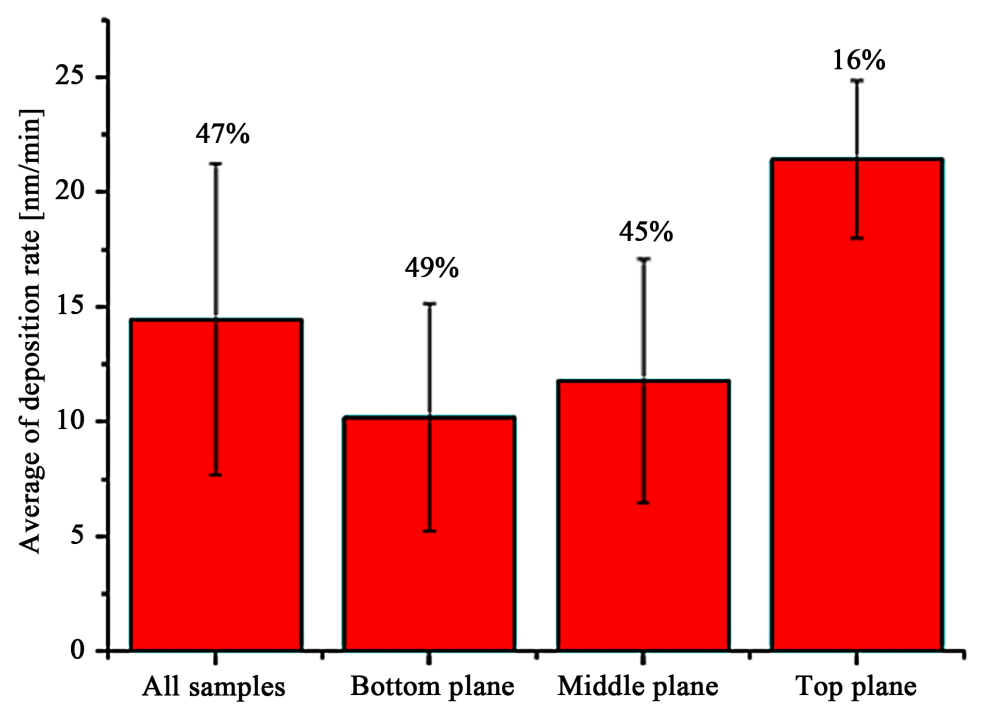

Figure 7. Average of deposition rate-Experiment current setup. 
- The gas flow plays a role in the homogeneity of the coating deposition rate.

- The gas feed-in system is apparently not influencing the coating deposition rate for the current setup.

\section{Part II: Solution to Minimize the Influence of the Gas Exhaustion System}

With the aim of verify the previous hypotheses, it was necessary to create new solutions to modify the gas flow inside the chamber by reducing the influence of the gas exhaustion system. In this context, three eligible configurations were considered in respect to the desired requirements (Figure 8). The red " $\mathrm{x}$ " means out of requirement and the green mark means that the requirement is satisfied. Mandatory is a configuration that allows very homogeneous gas exhaustion from the chamber along with applicability in plasma coating process. If these two requirements can be fulfilled together with versatility, low construction cost and short construction time then it would be the optimal one. The standard condition of the plasma-coater is a large (260 $\mathrm{mm}$ diameter) central exhaustion flange with a perforated metallic grid installed under it. One idea is to substitute this single flange by more flanges and distribute them in an organized way on the top of the plasma chamber. This construction can homogenize the gas exhaustion once the low pressure inside the plasma chamber is taken into consideration. This is a different situation from the gas feed-in where inside the pipe a high pressure is generated [15]. It means that the dimensions of the flanges must be carefully designed so as to reduce the pressure drop and not to jeopardize the vacuum pump capacity. However this solution offers very low versatility, high construction cost and high construction time. Other alternative evaluated was the installation of perforated pipes (without the bottom surface) between the perforated metallic grid and the central exhaustion flange. These kinds of pipes would be manufactured in a plate with the aim to let the gas exhaustion just through the pipe holes. This solution can achieve homogeneous gas exhaustion as well with relative low construction cost. Nevertheless, it requires time to construct and it is not versatile. Any future change would imply in time and money cost. Lastly, a solution with a thin perforated plate installed over the perforated

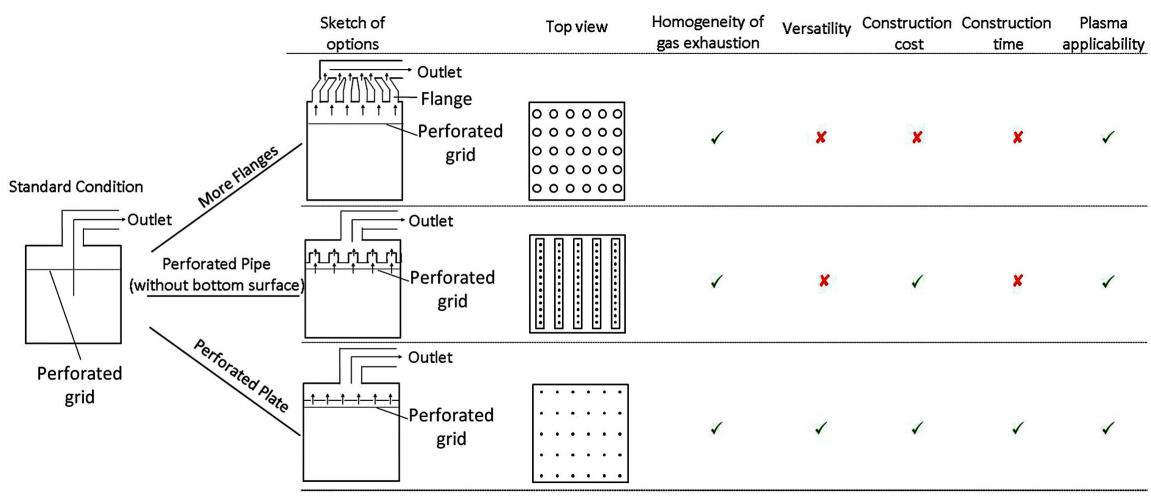

Figure 8. Eligible gas exhaustion configurations and desired requirements. 
metallic grid was evaluated and it satisfies all the defined requirements. As the plate is thin, the pressure drop will not be significant which allows a larger range of holes diameter. Therefore, the hole diameter can be tailored taking into consideration the vacuum pumping capacity to achieve the desired working pressure. Moreover, this configuration allows quick modifications, has low cost and short construction time. Therefore, this idea was selected to be developed in this work.

Through the development of gas feed-in systems, it was shown [15] that the pressure inside the gas feed-in pipe must be homogeneous over length and higher than the chamber pressure to achieve an even gas supply through the pipe holes. The same principle was applied with the aim to manipulate the gas exhaustion system. Basically, the gas must be removed evenly from the low-pressure chamber to allow a homogeneous gas flow distribution inside it.

In this context, a perforated plate was developed. It was named Baffle-Plate. It is similar to ideas used in other applications, i.e. control the fluid flow for instrumentation accuracy requirements [18]. The idea is to create comparable effect, like the installation of more flanges, but in a simpler and much more flexible manner from the construction point of view. This effect can be achieved by the Baffle-Plate where the holes will act as smaller "exhaustion systems" without huge modifications in the low-pressure plasma chamber and it avoids high investments. The challenge is to develop this Baffle-Plate in a way that all the holes will show the same pressure difference between the regions under and above the holes what will imply in homogenous gas exhaustion from each hole. Thereupon, it is very important to define the influence of the Baffle-Plate layout constructions on pressure loss through the holes. The constructor needs to take into consideration the desired work pressure for the plasma polymerization process and the pump suction capacity by developing the Baffle-Plate. This is very important since the control over the gas residence time, which can be achieved through the control over the working pressure by installation of the Baffle-Plate, can lead to powder formation by the plasma polymerization process, if the pressure is too high.

\subsection{Methods}

\subsubsection{CFD Simulations}

With the aim of create guidelines for the construction of Baffle-Plates, CFD simulations were performed in the same way as described for the current condition in Part I. Bayazit and coauthors [19] showed that for laminar flow regime the larger pressure drop occurred for larger plate thickness $(\mathrm{t} / \mathrm{D}=1)$, where " $\mathrm{t}$ " is the plate thickness and " $D$ " the hole diameter. Therefore, for this study a thin plate $(t=3 \mathrm{~mm}, \mathrm{t} / \mathrm{D}=0.17)$ was used to avoid high pressure drop. The starting point was the simulation of five distinct distances between the Baffle-Plate and the top of the chamber (Figure 9), keeping constant other reference parameters as presented in Table 2, the resulting curve was named reference curve. Afterwards, 


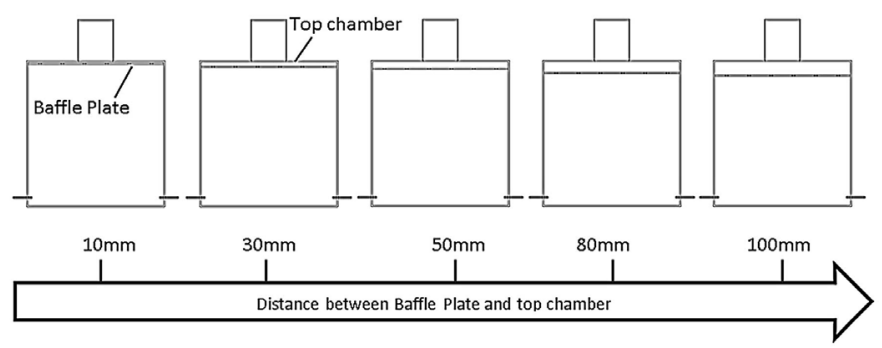

Figure 9. Representation of the low-pressure plasma chamber with different distances between the Baffle Plate and the top chamber.

Table 2. Baffle-Plate technical layout under simulation analysis.

\begin{tabular}{|c|c|c|c|c|c|}
\hline \multirow[b]{2}{*}{$\begin{array}{c}{ }^{*} \text { Distance } \\
\text { to the top } \\
{[\mathrm{mm}]}\end{array}$} & \multirow[b]{2}{*}{ Reference parameters } & \multicolumn{4}{|c|}{ Changed parameters } \\
\hline & & $\begin{array}{c}\text { Flow rate } \\
{[\mathrm{sccm}]}\end{array}$ & $\begin{array}{l}\text { Number } \\
\text { of holes }\end{array}$ & $\begin{array}{c}\text { Hole } \\
\text { Diameter } \\
{[\mathrm{mm}]}\end{array}$ & $\begin{array}{l}{ }^{* *} \text { Chamber } \\
\text { volume }\end{array}$ \\
\hline 107 & & & & & \\
\hline 30 & Flow rate $[\mathrm{sccm}] \rightarrow 145$ & 300 & - & - & - \\
\hline 50 & Number of holes $\rightarrow 30$ & - & 54 & - & - \\
\hline & & - & - & 30 & - \\
\hline $\left.\begin{array}{c}80 \\
100\end{array}\right]$ & Hole diameter $[\mathrm{mm}] \rightarrow 18$ & - & - & - & $2 / 3$ \\
\hline
\end{tabular}

${ }^{\star}$ Distance from the Baffle-Plate to the top of the low-pressure plasma chamber; ${ }^{*}$ Two thirds of the original model volume were used.

the CFD simulations were performed varying the values of some parameters as shown in Table 2 under the changed parameters column. For each simulation one parameter was varied while the others were kept constant.

\subsubsection{Experiment}

With the intention to identify the practical influence of the Baffle-Plate in respect to the homogeneity of the coating deposition rate, the same experiment performed previously for the current condition was done. However, now with the Baffle-Plate installed as depicted in Figure 10.

The Baffle-Plate lay-out should take into consideration the pressure difference between the upper and the lower region of the plate. This pressure difference must exist in order to be possible to remove the gas from the chamber in an even way. In the concrete case the measured pressure difference is $0.01 \mathrm{hPa}$. The hole diameter must be in the order of $15 \mathrm{~mm}$ and it is direct related to the pump suction capacity. If very big hole diameter is employed, for instance $30 \mathrm{~mm}$, the effect of the Baffle-Plate will be reduced due to the increase of the influence zone of each hole inside the chamber as well as due to a huge reduce in the pressure difference. Therefore it is recommended to start the construction with small holes and distribute them in an organized manner. For the current work, 30 holes with $18 \mathrm{~mm}$ diameter distributed in 5 columns with 6 holes each one is used. The idea is to cover properly the chamber section area allowing the suction 


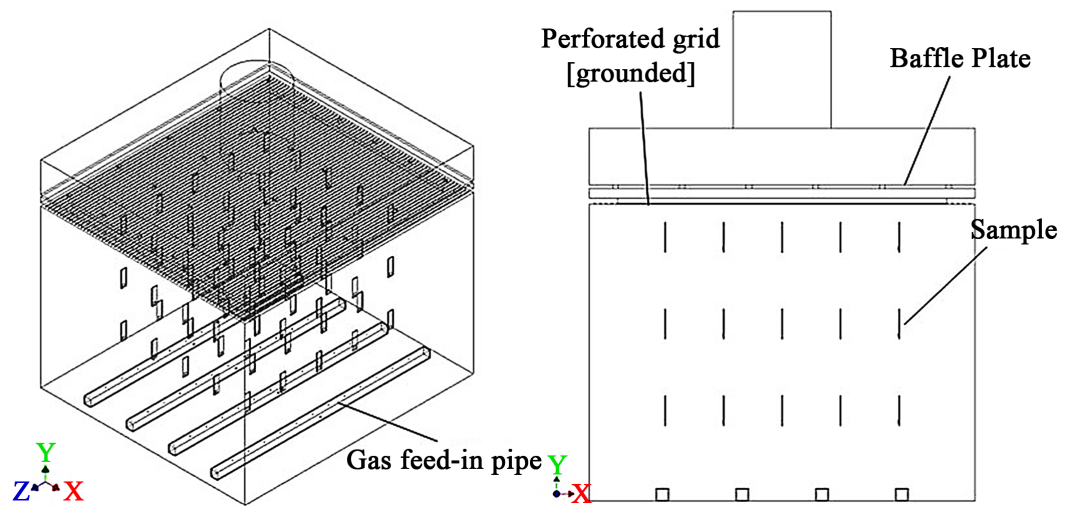

Figure 10. Scheme of the experiment setup with the Baffle-Plate installed.

of the gas evenly instead to concentrate it in few positions. After the Baffle-Plate being installed, pressure measurements must be done over and under it to check if the desired working pressure is reached. In case the working pressure is higher than the desired one, the diameter can be enlarged and the pressure measured again. Furthermore, it is very important to install the Baffle-Plate keeping a distance from the perforated grid. If the Baffle-Plate is installed direct in contact with the perforated grid the pressure loss would drastically increase, increasing the work pressure, requiring more pump efforts to achieve the desired pressure. The distance between Baffle-Plate and perforated grid applied in this work was $25 \mathrm{~mm}$.

\subsection{Results and Discussion}

\subsubsection{CFD Simulations}

Due to the installation of the Baffle-Plate is expected that the coating deposition rate will be more homogeneously distributed inside the chamber thanks to the achieved even gas flow as exhibited in Figure 11. In all three planes the gas velocity over most of the area is homogeneous $(\sim 0.2 \mathrm{~m} / \mathrm{s})$. Close to the walls and samples the gas velocity is restrained, tending to $0 \mathrm{~m} / \mathrm{s}$.

Figure 12 show the calculated non-uniformity coefficient for the five distinct distances between the Baffle-Plate and the top of the chamber. The non-uniformity coefficient was calculated in the same way as presented in "Part I: Current condition". It can be observed that the non-uniformity coefficient increases slightly from $100 \mathrm{~mm}$ to $50 \mathrm{~mm}$ distance and from $50 \mathrm{~mm}$ toward $10 \mathrm{~mm}$ it increases reasonably. This scenario is quite similar to the gas feed-in pipe construction described in our previous work [15], where lower pipe section area led to higher non-uniformity coefficient.

Figure 13 displays the velocity and pressure plot contour for two different distances between Baffle-Plate and top of the chamber. Left side shows the plots for $100 \mathrm{~mm}$ distance and right side for $10 \mathrm{~mm}$ distance. Comparing the two pressure contour plots, by $100 \mathrm{~mm}$ (top left) there is no pressure gradient (overall blue) over the Baffle-Plate unlike the case with $10 \mathrm{~mm}$ distance (top right) where a gradient is seen represented by the transition from green color on 


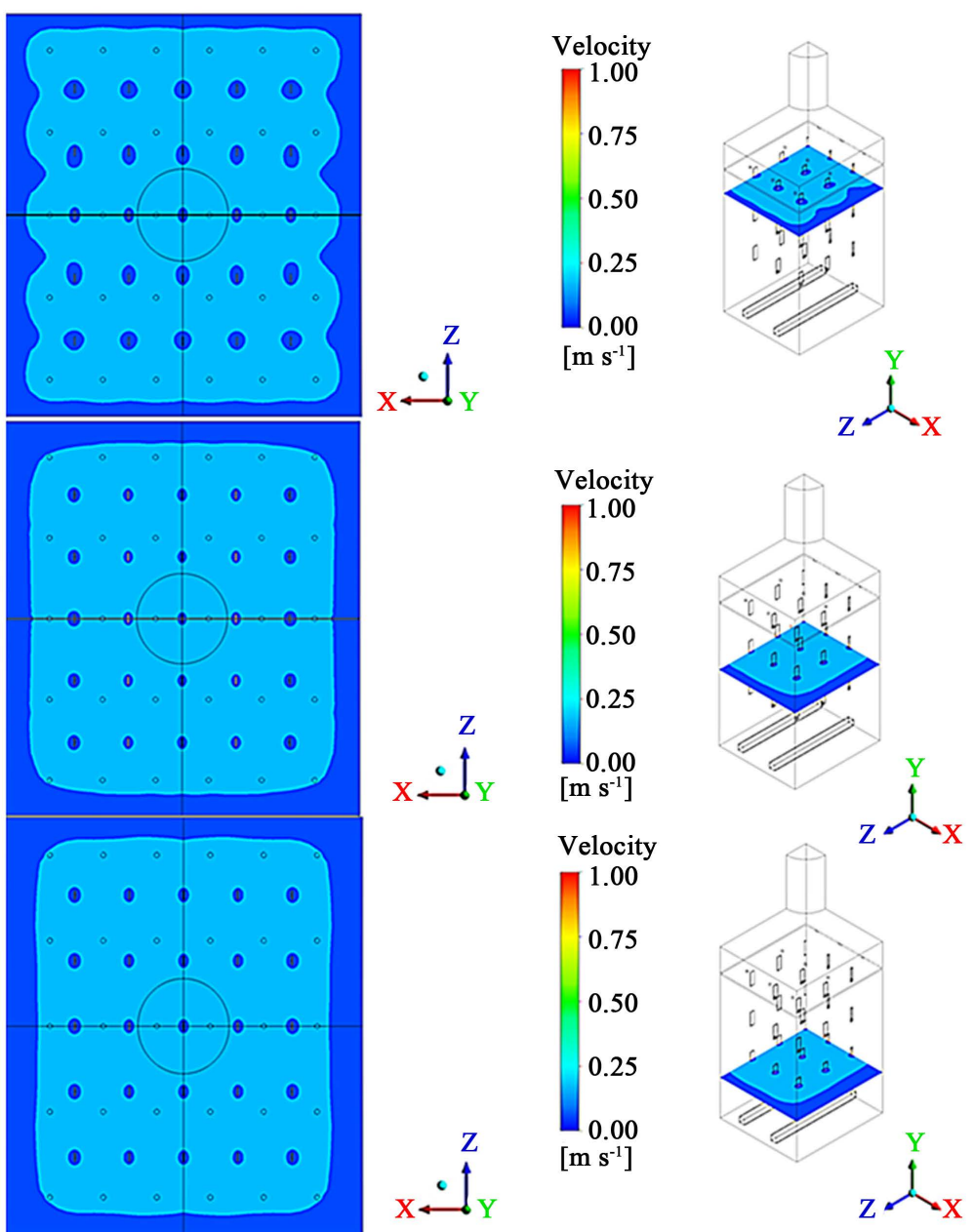

Figure 11. Gas simulation results of the experiment-Current setup with Baffle-Plate installed.

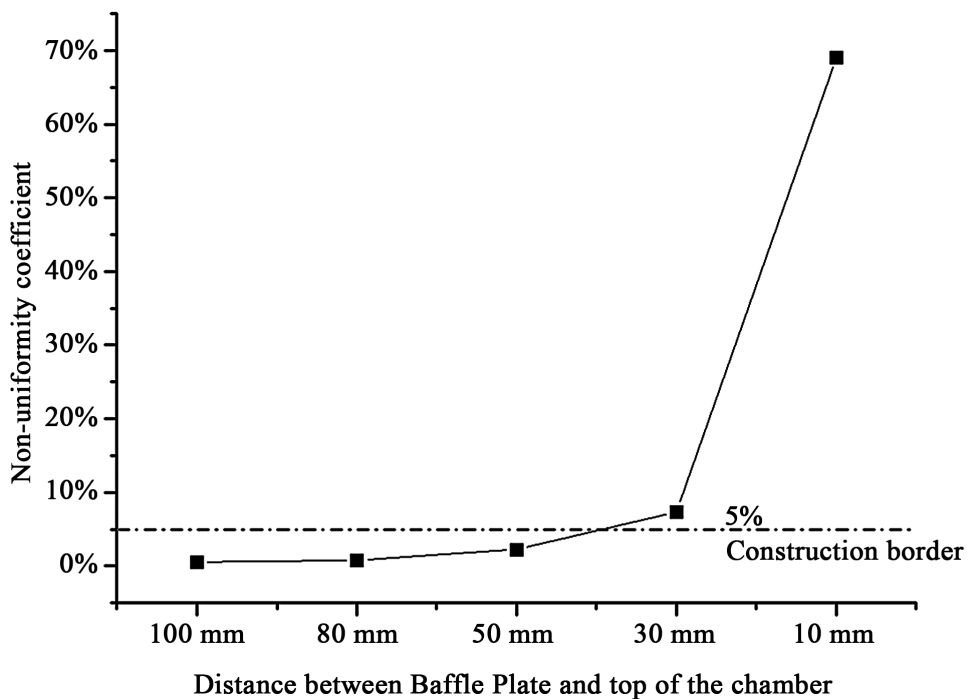

Figure 12. Influence of the distance from the Baffle-Plate to the top of the chamber on the non-uniformity coefficient. The results are shown via the points; the lines connecting the points in the plot are only to support guiding the eyes and are not modelled fits. 


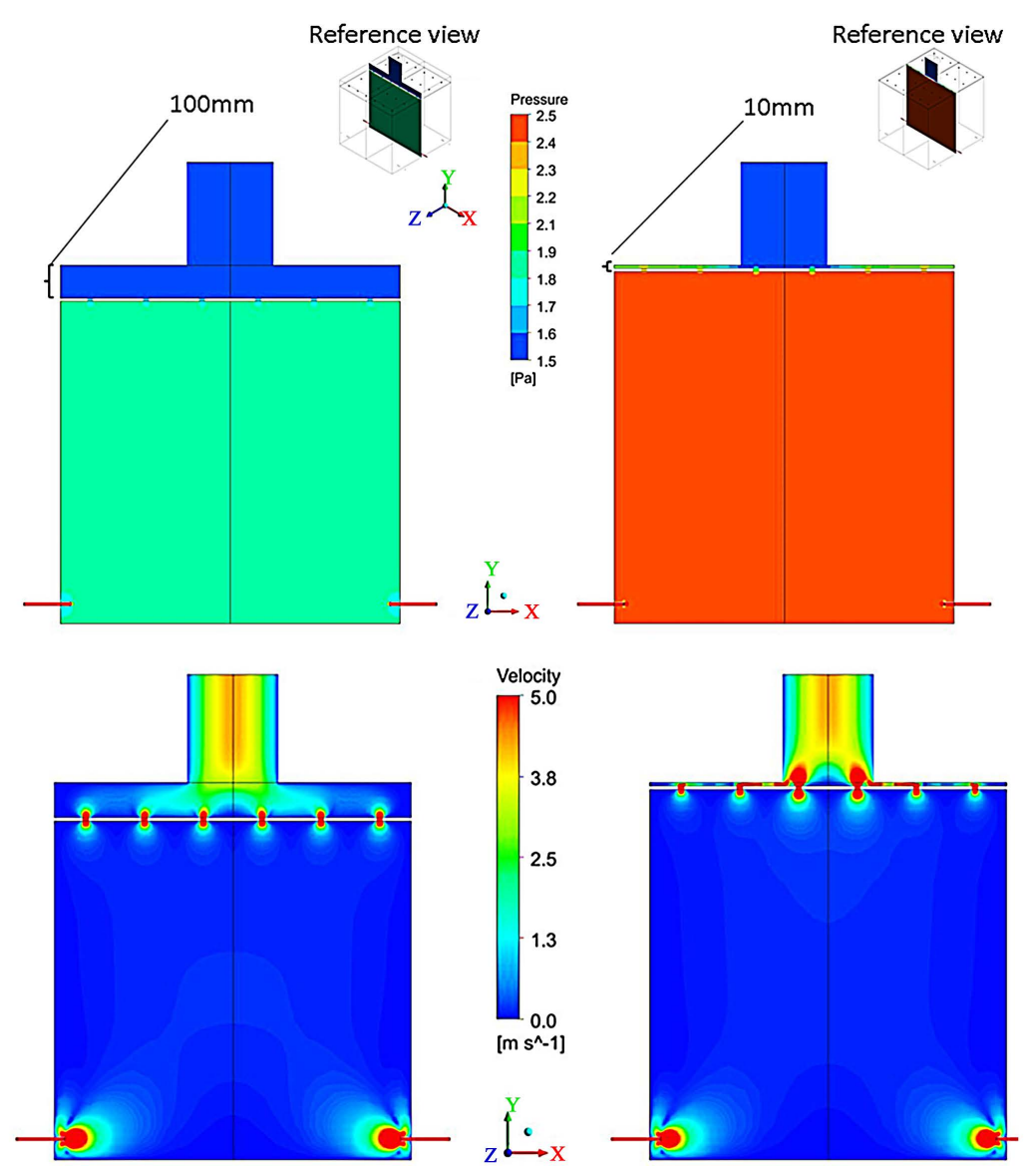

Figure 13. Gas flow simulation results showing pressure contour and velocity contour for two different distances between Baffle-Plate and top of the chamber.

the sides to blue color in the middle. Due to this pressure gradient, it can be observed in the velocity plot contour for $10 \mathrm{~mm}$ distance case (bottom right) that the gas velocity is higher in the middle holes in comparison to the holes localized in the sides. For the case with $100 \mathrm{~mm}$ distance (bottom left) the gas velocity is the same in all exhibited holes.

\subsubsection{Experiment}

With the intention to compare the coating deposition rate homogeneity, obtained through the experiment with current setup and through the experiment with the Baffle-Plate installed, both color contour graphic of the distribution of the calculated deposition rate are shown in Figure 14. The results are divided in three planes: top plane (Figure 14(a)), middle plane (Figure 14(b)) and bottom plane (Figure 14(c)). Each plane contains 25 samples whose positions are represented by the black dot points in each image. The results were organized in two columns where the left one represents the results without Baffle-Plate and the right one with the Baffle-Plate installed. All images were generated using the same scale bar, varying from $3 \mathrm{~nm} / \mathrm{min}$ to $30 \mathrm{~nm} / \mathrm{min}$.

Figure 14 shows that the deposition rates are still higher close to the walls in comparison to the chamber centre. Nevertheless, the color gradient in the right 


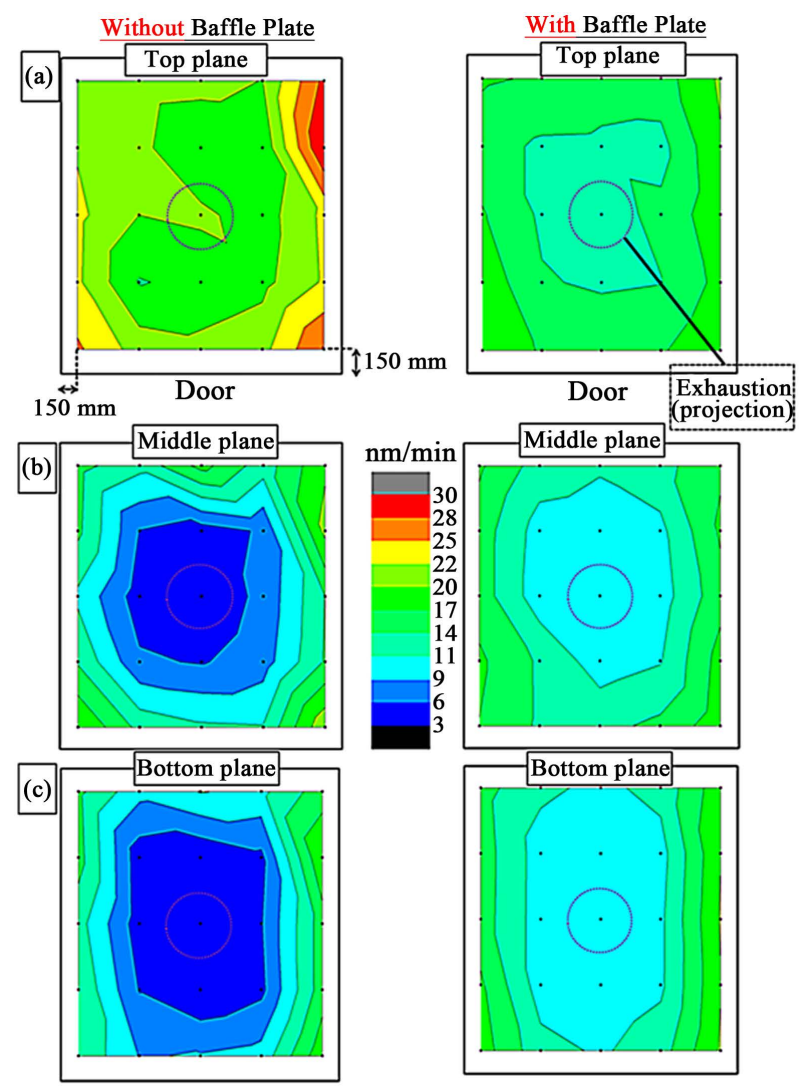

Figure 14. Comparative of coating deposition rate with and without the Baffle-Plate installed.

column, which represents the experiment with the Baffle-Plate installed, are weaker than the color gradient in the left column (without the Baffle-Plate). Like in the experiment without the Baffle-Plate, the images from the right column (with Baffle-Plate) show also higher deposition rate in average (dark green) in the top plane in comparison to the bottom and middle plane. Nonetheless, this difference is not as big as in the case without the Baffle-Plate as showed in Figure 15. The improvement in the coating homogeneity due to the installation of the Baffle-Plate is clearly visible in Figure 15. It shows a reduction in $50 \%$ of the standard deviation for the process with Baffle-Plate installed (23\%) in comparison to the previous one without Baffle-Plate installed (47\%) considering all samples. Moreover, the average of deposition rates considering all 75 samples in both experiments were the same. It gives a hint that the Baffle-Plate do not influence the deposition rate itself, in fact just work in a way to homogenize it.

Regarding the properties of the obtained coating, Figure 16 shows that both experiments exhibited a similar surface energy in average. The inhomogeneity $(\Phi)$ in both experiments is low and in the range of $5 \%-8 \%$. Additionally, there are no big differences among all three planes. As the surface energy is related to the chemical composition of the coating, it indicates that by the installation of the Baffle-Plate no influence was generated in a way that could lead to different coating chemistry in different regions, which is an expected and positive signal. 


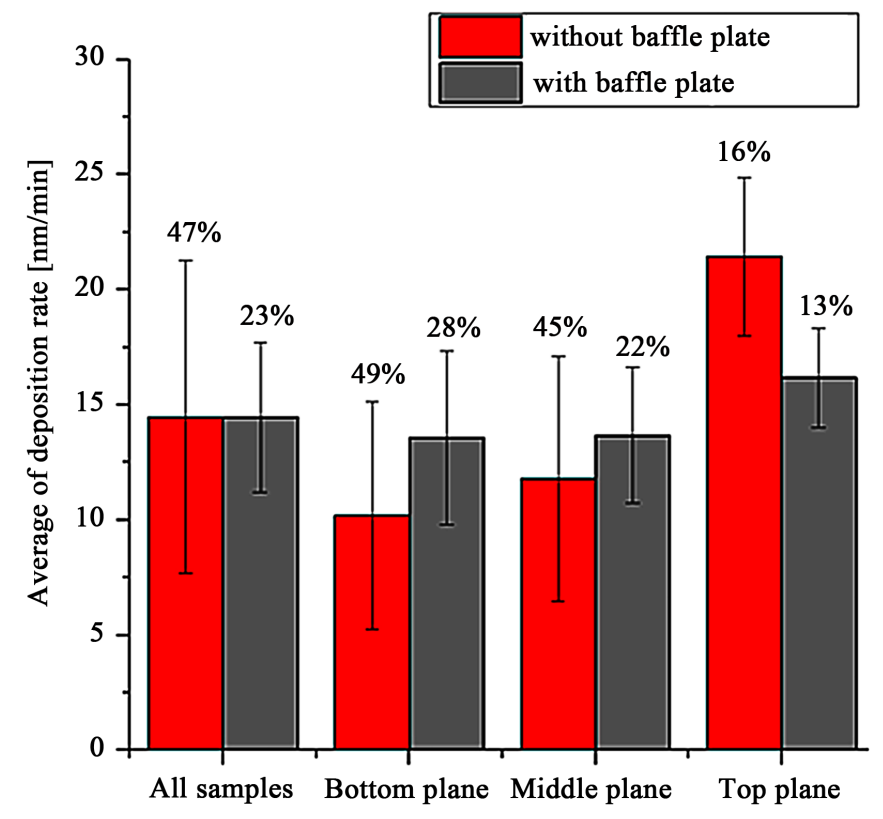

Figure 15. Comparative of the average of deposition rate-Experiment current setup and with Baffle-Plate installed.

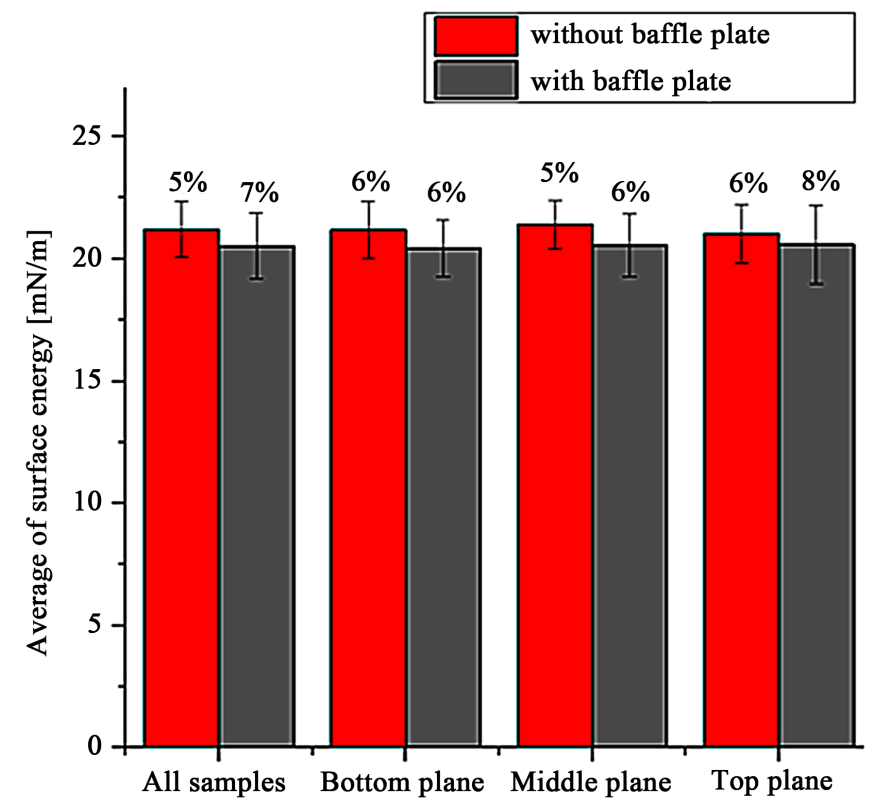

Figure 16. Comparative average of the surface energy [Wu method] —With and without Baffle-Plate.

The results suggest that the use of aBaffle-Plate do not influence the properties of the coating (surface energy), but it increases the homogeneity of the coating deposition rate since the only difference between both experiments was the change in the gas flow behavior due to its installation. Nevertheless, the deposition rate pattern does not match exactly with the gas flow pattern calculated through simulation and showed previously in Figure 11. Therefore, additional investigations are required, not regarding the gas flow but the electric field. 


\section{Conclusions}

To understand the influence of the gas exhaustion system inside a $1 \mathrm{~m}^{3}$ low-pressure plasma chamber on the homogeneity of the coating deposition rate, gas flow simulations along with practical coating experiments are performed. From the outcomes obtained through initial investigation with the current setup of the plasma coater, a solution to homogenize the gas exhaustion system is developed and described. The solution named Baffle-Plate, consists of a perforated plate, which is mounted underneath the gas exhaustion system. Thereby relevant constructions parameters are identified and investigated with the aim of understand their influence in the homogeneity of the gas exhaustion. Number of holes, hole diameter, distance from the Baffle-Plate to the top of the chamber, gas flow and chamber volume are the evaluated parameters.

The main conclusions extracted from the results are:

1) From the analyzed parameters the distance between Baffle-Plate and top chamber was the most relevant one influencing the homogeneity of gas exhaustion.

2) By decreasing the distance between Baffle-Plate and top chamber, the pressure gradient in this region increases resulting in nonhomogeneous gas exhaustion through the holes. For instance, the nonuniformity coefficient for $100 \mathrm{~mm}$ distance is $0.5 \%$ and for $10 \mathrm{~mm}$ distance is $69 \%$.

3) Taking the construction border $(\Phi<5 \%)$ into consideration the results showed that the installation of the Baffle-Plate with a distance above $80 \mathrm{~mm}$ to the top chamber is in a safety area regarding the homogeneity of gas exhaustion for low-pressure application.

4) From the analyzed values, flow rate and number of holes do not have a considerable influence in the homogeneity of gas exhaustion through the holes. However, it is recommended to start the layout with hole diameter in the order of $15 \mathrm{~mm}$.

5) The hole diameter strongly influences the homogeneity of gas exhaustion through the holes, especially with high distances between Baffle-Plate and top of the chamber.

6) The chamber volume has an influence in the homogeneity of gas exhaustion, especially with high distances between Baffle-Plate and the top of the chamber.

7) The Baffle-Plate is an effective solution to improve the homogeneity of coating deposition rate for plasma polymerization processes.

In this investigation it was not possible to run the gas flow simulations with the metallic perforated grid installed due to limited computer processing capacity. Nevertheless, for this work, the simulations results are still valuable once they allow to predict the gas velocity distribution and consequently to have hints about the distribution of the coating deposition rate inside the plasma coater.

The layout and the installation of a Baffle-Plate for the described plasma-coater showed in practical coating experiments that the non-uniformity 
coefficient was enhanced from $47 \%$ (without Baffle-Plate) to $23 \%$. Low values from the nonuniformity coefficient mean more homogeneous coating distribution.

The construction guidelines for the use of a Baffle-Plate solution for large volume PECVD chambers developed in this work showed to be very effective in terms of achieve homogenous coating deposition rate. Basically, the person in charge should start with hole diameter in the order of $15 \mathrm{~mm}$ and distribute them in a proper manner aiming to avoid concentration of gas suction in small areas. Optimal is to distribute the holes in a way to cover the section area of the chamber. Furthermore, it is important to keep a distance, for instance $25 \mathrm{~mm}$, between the Baffle-Plate and the perforated grid to reduce pressure loss through the holes. After done the first layout, the next step is to assemble the Baffle-Plate and measure the pressure difference. If the desired working pressure is not reached, then the holes can be enlarged and proceed with measurements again until the desired working pressure is reached.

Like for the gas feed-in system presented in our previous work [15], the support of CFD tool was very successful to understand the influence of the gas exhaustion system, to study and to propose solution to enhance the homogeneity of coating deposition.

\section{Acknowledgements}

The authors are grateful to the Science without Borders programme (Ciência sem Fronteiras, Gustavo Simiema de Freitas Barbosa 201387/2014-0) and the Brazilian National Council of Technological and Scientific Development (CNPq) for the financial support.

\section{References}

[1] Ohring, M. (1992) The Materials Science of Thin Films. Academic Press, San Diego.

[2] Yasuda, H. (1985) Plasma Polymerization. Academic Press, Inc., Orlando.

[3] Bewilogua, K., Bräuer, G., Dietz, A., Gäbler, J., Goch, G., Karpuschewski, B. and Szyszka, B. (2009) Surface Technology for Automotive Engineering. CIRP Annals-Manufacturing Technology, 58, 608-627.

https://doi.org/10.1016/j.cirp.2009.09.001

[4] Paulkowski, D., Karpinski, S. and Vissing, K. (2014) Friction and Wear Resistance of Plasma Polymeric Coatings Applied on Elastomers. Tribologia: Finnish Journal of Tribology, 32, 12-19.

[5] Martinu, L. and Poitras, D. (2000) Plasma Deposition of Optical Films and Coatings: A Review. Journal of Vacuum Science \& Technology A Vacuum Surfaces and Films, 18, 2619-2645. https://doi.org/10.1116/1.1314395

[6] Petit-Etienne, C., Tatoulian, M., Mabille, I., Sutter, E. and Arefi-Khonsari, F. (2007) Deposition of $\mathrm{SiO}_{\mathrm{x}}$-Like Thin Films from a Mixture of HMDSO and Oxygen by Low Pressure and DBD Discharges to Improve the Corrosion Behaviour of Steel. Plasma Processes and Polymers, 4, S562-S567. https://doi.org/10.1002/ppap.200731406

[7] Kim, Y.T., Min, S.-K., Hong, J.S. and Kim, C.-K. (1991) Highly Conductive Tungsten Thin Films Prepared by the Plasma-Assisted Silane Reduction Process. 
Japanese Journal of Applied Physics, 30, 820-826.

https://doi.org/10.1143/JJAP.30.820

[8] Vogel, K., Westphal, N., Salz, D., Thiel, K., Wittig, L., Ciacchi, L.C. and Grunwald, I. (2015) Dental Implants Coated with a Durable and Antibacterial Film. Surface Innovations, 3, 27-38. https://doi.org/10.1680/si.14.00002

[9] Paulkowski, D. and Vissing, K. (2011) Tribological Improvement of Elastomers Using Plasmapolymeric Coatings. Proceedings of Tribologie Fachtagung, Göttingen, 21-23 September 2009, Vol. 1, 15/1-15/14.

[10] Gürsoy, M. and Karaman, M. (2016) Hydrophobic Coating of Expanded Perlite Particles by Plasma Polymerization. Chemical Engineering Journal, 284, 343-350. https://doi.org/10.1016/j.cej.2015.09.007

[11] Herchen, H., Merry, W. and Brown, W. (1998) Etch Enhancement Using an Improved Gas Distribution Plate.

[12] Jeong, K.C. (1998) Gas Distribution System and Method for Chemical Vapor Deposition Apparatus.

[13] Hanawa, H., Maung, K. and Chung, H. (2011) Gas Distribution Showerhead and Method of Cleaning.

[14] Su, Y.-J. (1996) Gas Distribution Plate for Semiconductor Wafer Processing Apparatus with Means for Inhibiting Arcing.

[15] Barbosa, G.S.F., Vissing, K. and Mayer, B. (2016) Creation and Evaluation of Construction Guidelines Using CFD for Low Pressure Plasma Gas Feed-In Systems to Homogenize the Precursor Gas Flow. OJFD, 6, 391-405. https://doi.org/10.4236/ojfd.2016.64029

[16] Giessler, S., Just, E. and Störger, R. (2006) Easy-to-Clean Properties-Just a Temporary Appearance? Thin Solid Films, 502, 252-256.

https://doi.org/10.1016/j.tsf.2005.07.292

[17] Brenner, T. (2017) Struktureigenschaftsbeziehung von chemisch gleichartigen plasmapolymeren Trennschichten mit stark variierendem Elastizitätsmodul. Master Thesis.

[18] Malavasi, S., Messa, G., Fratino, U. and Pagano, A. (2012) On the Pressure Losses through Perforated Plates. Flow Measurement and Instrumentation, 28, 57-66. https://doi.org/10.1016/j.flowmeasinst.2012.07.006

[19] Bayazit, Y., Sparrow, E.M. and Joseph, D.D. (2014) Perforated Plates for Fluid Management: Plate Geometry Effects and Flow Regimes. International Journal of Thermal Sciences, 85, 104-111. https://doi.org/10.1016/j.ijthermalsci.2014.06.002 